\title{
A Virtually Continuous Representation of the Deep Structure of Scale-Space
}

\author{
Luigi Rocca and Enrico Puppo \\ Department of Informatics, Bio-engineering, Robotics and System Engineering \\ University of Genova - Via Dodecaneso 35 - 16146 Genova - Italy \\ \{rocca, puppo\}@dibris.unige.it
}

\begin{abstract}
The deep structure of scale-space of a signal refers to tracking the zero-crossings of differential invariants across scales. In classical approaches, feature tracking is performed by neighbor search between consecutive levels of a discrete collection of scales. Such an approach is prone to noise and tracking errors and provides just a coarse representation of the deep structure. We propose a new approach that allows us to construct a virtually continuous scale-space for scalar functions, supporting reliable tracking and a fine representation of the deep structure of their critical points. Our approach is based on a piecewise-linear approximation of the scale-space, in both space and scale dimensions. We present results on terrain data and range images.
\end{abstract}

Keywords: scale-space, multi-scale analysis, topology of scalar fields.

\section{Introduction}

Since seminal works of Witkin [12] and Koenderink [8], scale-space methods have been greatly studied in the literature on computer vision and image processing - see [10] Section 4.4 for un updated bibliography - and later on in scientific visualization 12677. The deep structure of the scale-space 9] captures the evolution of differential properties across scales, by tracking zero-crossings of differential invariants - most typically, the critical points of a scalar field. The importance of critical points is measured by their life time in the scale-space and helps identifying relevant features that subsume the structure of the signal.

The standard approach to scale-space is discrete: a scale-space consists of a collection $\left(f_{0}, \ldots, f_{k}\right)$ of subsequently filtered versions of an input signal $f=f_{0}$. Feature points are identified in each level $f_{i}$ and the deep structure is extracted by tracking each feature across pairwise consecutive signals $f_{i}, f_{i+1}$ : given a feature point in $f_{i+1}$, a neighborhood of its location is explored in $f_{i}$, searching for a feature of the same type; feature points of $f_{i}$ that do not match any feature point in $f_{i+1}$ are considered to end their life at scale $i$; etc. Such a procedure is prone to false and missed matchings, and the matching process is greatly influenced by the granularity of sampling through scales.

In [11, a method was introduced to track the critical points of a scalar field, which follows the gradient of the field to detect correspondences across levels of

A. Petrosino (Ed.): ICIAP 2013, Part II, LNCS 8157, pp. 522-531, 2013.

(C) Springer-Verlag Berlin Heidelberg 2013 
the scale-space. Albeit more robust than standard neighbor search, this approach is still discrete, as the analysis is again performed at the level of granularity of sampling in the scale dimension, with similar drawbacks.

In this work, we propose a new approach that provides a virtually continuous model of the scale-space and of its deep structure. We adopt a piecewise-linear representation of the input signal $f$, by discretizing its domain with a simplicial mesh (a triangle mesh for a bi-variate function) having vertices at the sample points. The connectivity structure of samples provides a straightforward local criterion to detect critical points. We also adopt a piecewise-linear representation of the diffusion flow that generates the scale-space. Given the collection of snapshots $\left(f_{0}, \ldots, f_{k}\right)$, we assume the flow to be linear between each pair $f_{i}$, $f_{i+1}$. This allows us to easily track critical points across scales, because changes in the deep structure can occur only at a finite set of events, which are easy to find. The granularity of such events is much finer than sampling of snapshots. Tracking of critical points is exact in the context of this piecewise-linear approximation, while density of sampling in the scale dimension is just relevant to the approximation of diffusion flow, which is a non-linear process.

The trajectory of each critical point in the deep structure is encoded as a chain of edges of a mesh discretizing the domain of $f$; in this model, velocity of displacement of each critical point along its trajectory is piecewise constant and it is encoded by storing times of transitions through vertices of the mesh on a continuous scale; bifurcations, corresponding to deletion/creation of pairs of critical points, occur at midpoints of edges of the mesh where trajectories end/start. With this data structure at hand, it is straightforward to perform a number of queries about the scale-space and its deep structure, such as: slicing the scale-space at any arbitrary scale; evaluating and visualizing the trajectories of all critical points; selecting critical points according to their life span; etc.

\section{Scale-Space}

For the sake of simplicity, in the following we will deal just with the linear scalespace of a bi-variate scalar function defined on a 2D rectangular domain and sampled on a regular grid (such as images and digital elevation models). Most concepts introduced in this article, however, can be generalized in a straightforward way to any kind of scale-space, for functions defined over general manifold domains, in higher dimensions, and with irregular sampling. Generalizations will be briefly discussed in Section 4 .

Let $f: \mathbb{R}^{2} \rightarrow \mathbb{R}$ be a bi-variate scalar function with bounded range. For convenience, we assume that $f$ is a Morse function, i.e., all critical points of $f$ are isolated. The linear scale-space $F_{f}(x, y, t)$ of $f$ is defined as the solution of the heat equation

$$
\frac{\partial}{\partial t} F_{f}=\alpha \Delta F_{f}
$$

with initial condition $F_{f}(x, y, 0)=f(x, y)$, where $\Delta$ denotes the Laplace operator (with respect to the space variables $x, y$ ) and $\alpha$ is a constant term tuning the 


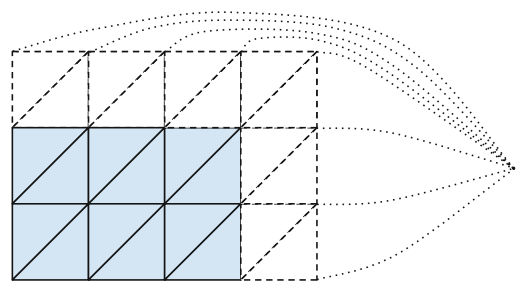

Fig. 1. Piecewise linear discretization of the domain (light blue triangles). Virtual points in the outer border (white triangles, dashed lines) correspond to a single point "at infinity" that completes the domain to a sphere-like topology.

speed of diffusion. So, scale-space is defined on a three dimensional domain, the first two dimensions referring to space. We will use interchangeably the words scale and time referring to the third dimension, as time comes from physical interpretation of the heat equation. Function $F_{f}$ can be obtained either through a diffusion process starting at $f$, or, equivalently, by filtering $f$ with Gaussian kernels of increasing variance, where variance is directly proportional to t.

Let $p$ be a critical point of $f$, i.e., a maximum, or a minimum, or a saddle. Generally speaking, point $p$ is displaced by the diffusion process, i.e.: it will possibly change its position for different values of $t$; and eventually it may disappear by collapsing with another critical point $p^{\prime}$. Collapses always involve critical points of different types, the only possibilities being either minimum-saddle, or maximum-saddle. In linear scale-space - as well as in most other existing scalespaces - critical points may also appear in pairs at a time $t>0$, with the same pairing rules. The tracking of a critical point $p$ through its life in scale-space provides a trajectory, which is a continuos line in the $2 \mathrm{D}+$ time domain. The trajectory starts either at $t=0$, or at time of birth of $p$; and it either ends at time of collapse, or it extends to infinity. The life span of $p$ is the interval spanned by the trajectory in time dimension, which provides a measure of how relevant $p$ is across the different scales.

In a discrete representation, scale-space is sampled at a finite set of times $\left(t_{0}=0, t_{1}, \cdots, t_{k}\right)$ on a bounded domain $D$, which we will assume to be a rectangle. A straightforward discrete representation consists of a collection of snapshots $\left(f_{0}, \ldots, f_{k}\right)$, where each snapshot $f_{i}$ is a discrete sampling of $F_{f}\left(\cdot, \cdot, t_{i}\right)$ at the nodes of a regular $m \times n$ grid $G_{D}$ over domain $D$, i.e., $f_{i}=\left\{f_{i}[r, c] \mid r=\right.$ $1, \ldots, m, c=1, \ldots, n\}$. If $p$ is a node of $G_{D}$ at coordinates $[r, c]$, we will use the shorthand $f_{i}(p)$ to denote $f_{i}[r, c]$.

\subsection{Piecewise-Linear Discretization of Space}

We adopt a piecewise-linear representation of $D$ by subdividing it into triangles as follows: each sampling point of coordinates $[r, c]$ is connected to its neighbors to form triangles $([r, c],[r, c+1],[r+1, c+1])$ and $([r, c],[r+1, c+1],[r+1, c])$, as depicted in Figure 1. We extend the domain with a virtual outer border, 
imposing the value of all samples of $f_{i}$ in the outer border to be an arbitrarily large negative value (virtually, $-\infty$ ). In this way, all sampling points have exactly six neighbors; the extended domain can be seen as having the topology of a sphere (where all nodes in the outer border collapse to a single point); and the function has a global minimum in the outer border, at all scales.

A discrete function sampled at the nodes of $G_{D}$ is thus extended to a piecewiselinear function interpolating the samples. With abuse of notation, in the following we will use the same symbols $f_{0}, \ldots, f_{k}$ to denote the piecewise-linear models of the sampled functions in the scale-space. Critical points of a function can occur only at sampling points:

- A minimum/maximum is a sample whose value is smaller/larger than the values of all its neighbors;

- A saddle is a sample such that, when its neighbors are traversed in cyclic radial order, the number $s$ of times that their values alternate between smaller and larger values with respect to the sample itself is larger than two. The index of the saddle is $s / 2-1$ : since each sampling point has exactly six neighbors, only 1- and 2-saddles may exist.

Note that in the smooth case, a Morse function admits just 1-saddles. Multiple saddles that arise in the piecewise-linear model correspond to different 1-saddles collapsing to the same point, and so they will be treated in our model. The Poincaré-Hopf index theorem guarantees that $n_{M}+n_{m}-n_{s}=1$, where $n_{M}$ is the number of maxima, $n_{m}$ is the number of minima (excluding the virtual minimum in the outer border), and $n_{s}$ is the number of saddles (where each 2 -saddle counts for two). Also note that a classical model based on either 4or 8-connectedness of pixels would not provide a classification of critical points consistent with the theorem, thus hindering the correct pairwise simplification of critical points through scales (see Section 2.3).

\subsection{Piecewise-Linear Discretization of Time}

Given the piecewise-linear snapshots $f_{0}, \ldots, f_{k}$ as defined above, let $p=[r, c]$ be a node of $G_{D}$. We define a piecewise-linear approximation of $F_{f}(r, c, \cdot)$ interpolating all samples $f_{0}(p), \ldots, f_{k}(p)$. In this way, we may slice the scale-space in the continuum, at any time $t \in\left[t_{0}, t_{k}\right]$, obtaining a new snapshot $f_{t}$ that is again a piecewise-linear function defined on the same triangular tiling of $D$. Let $i$ be such that $t_{i}<t \leq t_{i+1}$, by linear interpolation we have

$$
f_{t}(p)=\frac{t_{i+1}-t}{t_{i+1}-t_{i}} f_{i}(p)+\frac{t-t_{i}}{t_{i+1}-t_{i}} f_{i+1}(p) .
$$

Combined use of piecewise-linear approximations in both space and time provides an approximated model $\tilde{F}_{f}$ of $F_{f}$ that is continuous in the domain $D \times\left[t_{0}, t_{k}\right]$. Because of its piecewise-linear structure, it is possible to analyze the deep structure of $\tilde{F}_{f}$ in an exact way, as we will show in the following. 
Table 1. Label transitions at a flipping edge $(a, b)$ : a transition $l_{a} l_{b} \rightarrow l_{a}^{\prime} l_{b}^{\prime}$ means that $a$ has label $l_{a}$ before the flip and label $l_{a}^{\prime}$ after it, and similarly for $b$. Symmetric transitions and transitions involving maxima, which are analogous to those involving minima, are not listed for brevity.

\begin{tabular}{|c|c|}
\hline Transition & Effect \\
\hline$l_{a} l_{b} \rightarrow l_{a} l_{b}$ & no change \\
\hline$m r \rightarrow r m$ & a minimum moves from $a$ to $b$ \\
\hline$s 1 r \rightarrow r s 1$ & a 1 -saddle moves from $a$ to $b$ \\
\hline$s 2 r \rightarrow r s 2$ & a 2-saddle moves from $a$ to $b$ \\
\hline$s 2 r \rightarrow s 1 s 1$ & a 2 -saddle splits into two 1-saddles \\
\hline$s 1 s 1 \rightarrow s 2 r$ & two 1 -saddles merge into one 2 -saddle \\
\hline$s 2 s 1 \rightarrow s 1 s 2$ & switch between a 2 -saddle and a 1-saddle \\
\hline$m s 1 \rightarrow r r$ & a minimum collapses with a 1 -saddle \\
\hline$m s 2 \rightarrow r s 1$ & a minimum collapses with a 2 -saddle, which becomes a 1 -saddle \\
\hline$r r \rightarrow m s 1$ & a minimum and a 1-saddle appear from two regular points \\
\hline$r s 1 \rightarrow m s 2$ & a minimum and a 2 -saddle appear from a regular point and a 1-saddle \\
\hline
\end{tabular}

\subsection{Tracking Critical Points}

Our approach to feature tracking is inspired to a mechanism first introduced in [4] for controlled-topology filtering. We introduce the following classification (labeling) for a node $p$ of $G_{D}$ :

- $\mathrm{m}: p$ is a minimum;

- M: $p$ is a maximum;

- s1: $p$ is a 1-saddle;

- s2: $p$ is a 2-saddle;

- $\mathrm{r}: p$ is a regular point (neither of the above).

We are interested in events that change the classification of $p$ during the diffusion process (i.e., while varying $t$ ). From the piecewise-linear model introduced in Section 2.1, it turns out that these events can occur only when two adjacent samples flip the order of their values. Let $a$ and $b$ be two adjacent nodes on the triangular tiling of $D$. We say that edge $(a, b)$ flips at time $t$ if and only if $f_{t}(a)=f_{t}(b)$ while for an arbitrarily small $\varepsilon>0$ we have either $f_{t-\varepsilon}(a)<f_{t-\varepsilon}(b)$ and $f_{t+\varepsilon}(a)>f_{t+\varepsilon}(b)$, or vice-versa. Let us consider two consecutive times $t_{i}$ and $t_{i+1}$ in the discrete time sequence. Since $\tilde{F}_{f}$ is linear between $t_{i}$ and $t_{i+1}$, edge $(a, b)$ flips in $\left[t_{i}, t_{i+1}\right]$ if and only if $f_{t_{i}}(a)<f_{t_{i}}(b)$ and $f_{t_{i+1}}(a)>f_{t_{i+1}}(b)$, or vice-versa. The exact time $t$ of flip is obtained by linear interpolation:

$$
t=\frac{\left(f_{i+1}(a)-f_{i+1}(b)\right) t_{i}+\left(f_{i}(b)-f_{i}(a)\right) t_{i+1}}{f_{i}(b)-f_{i}(a)+f_{i+1}(a)-f_{i+1}(b)} .
$$

We select all edges that flip in each interval $\left[t_{i}, t_{i+1}\right]$ and we sort them by time of flip. This sequence of flips provides all events that may cause changes in the 
deep structure. Given the flip of edge $(a, b)$ at time $t$ and given a labeling for $a$ and $b$ at time $t-\varepsilon$, the transitions described in Table 1 may occur.

We generate the deep structure of scale-space as follows. We evaluate all critical points of input signal $f$ and we initialize a data structure listing them all. The data structure is an array of lists: each entry in the array is aimed at recording the trajectory of a critical point $p$ through time, and it is linked from the corresponding entry of $p$ in the grid $G_{D}$; each entry in a list contains the location on $G_{D}$ of the corresponding critical point at a given time $t$. For each 2 -saddle we generate two entries in the array, accounting for the fact that two 1-saddles start at the same place and can proceed along the same trajectory until they possibly split, or one of them collapses.

For each $i=0, \ldots, k-1$, we sort by time all flips that occur in $\left[t_{i}, t_{i+1}\right]$. Then we scan the sequence of flips, and we update the trajectories and the corresponding links from $G_{D}$ according to relevant transitions (all except the first one in Table 1) that occur. A transition can be easily detected by comparing the height of $a$ and $b$ with all their neighbors just before and just after the time of split. Each transition that generates a pair of newborn critical points adds two new entries to the array of trajectories.

At the end of processing, the trajectory of each point consists of a chain of edges of the triangular tiling of $D$, with time stamps that record the "time of arrival" of the critical point at each node along the chain. For newborn critical points and for critical points that collapse we also record their pairing with the critical points that are born, or collapsed, together with them.

The array encoding trajectories will thus contain the lives of all critical points that appear in the deep structure, each encoded in a list. With a linear scan of the array, relevant points with respect to a given criterion can be extracted; the life span of each of them can be evaluated in constant time; while their position at any arbitrary time $t$ can be evaluated with a binary search of the list and a linear interpolation. This mechanism supports several queries: critical points alive at a given scale $t$ can be found without analyzing $f_{t}$, and they can be located either on $f_{t}$, or at their original position in $f$ (or at time of birth for newborn points); the trajectory of each critical point can be obtained; most relevant points can be extracted based on the length of their life, and they can be located in space at any scale during their life span; given an interval of scales $\left[t, t^{\prime}\right]$, points whose life span contains the given interval can be extracted; etc.

\section{$3 \quad$ Experimental Results}

We present results on two datasets: a $1200 \times 1200$ digital terrain model of a region around the Monte Rosa Massif in the Western Alps [3] and a $500 \times 500$ range image from the Texas 3D Face Recognition Database [5]. For each dataset, we have built a sequence of snapshots of the scale-space on an exponential scale, by applying cumulative Gaussian blur, starting at $\sigma=\sqrt{2}$ and doubling the scale at each level, up to scale 1024 for terrain , and up to scale 128 for the range image. In Table 2 we show statistics about the deep structure of the terrain dataset. 
Table 2. Statistics on terrain dataset. Left: number of critical points and average duration of their lives in linear and logarithmic scale; in brackets statistics on newborn critical points. Right: transitions (flips) in the scale-space that: do not change the deep structure; move a critical point; collapse a pair of critical points; generate a pair of critical points.

\begin{tabular}{|c|c|c|c|c|c|c|c|}
\hline scale & $\begin{array}{c}\text { \#Max } \\
\text { (newborn) }\end{array}$ & $\begin{array}{c}\text { \#min } \\
\text { (newborn) }\end{array}$ & $\begin{array}{c}\text { \#saddle } \\
\text { (newborn) }\end{array}$ & $\begin{array}{c}\text { avg life } \\
\text { (newborn) }\end{array}$ & $\begin{array}{c}\text { avg log life } \\
\text { (newborn) }\end{array}$ \\
\hline 3.0 & $1718(1164)$ & $1270(1100)$ & $2903(2201)$ & $1.40(0.61)$ & $10.34(2.32)$ \\
9.0 & $1171(903)$ & $906(863)$ & $2062(1705)$ & $1.34(0.58)$ & $12.94(2.41)$ \\
27.0 & $766(626)$ & $602(593)$ & $1347(1168)$ & $1.23(0.51)$ & $16.48(2.46)$ \\
81.0 & $401(336)$ & $285(285)$ & $680(596)$ & $1.25(0.48)$ & $23.94(2.65)$ \\
243.0 & $42(25)$ & $18(18)$ & $61(42)$ & $2.86(0.69)$ & $80.32(6.07)$ & no change & $1,100,422$ \\
move & 13,490 \\
collapse & 5,130 \\
birth & 2,579 \\
\hline
\end{tabular}

Note that, thanks to our data structure, we query scales different from those of the discrete sequence in input. On the left side, we report the number of critical points found at each scale, together with their average duration of life through scale-space, both on a linear scale (which tends to give longer lives to features appearing at large scales) and on a logarithmic scale (which better spreads the life spans through scales that grow exponentially). We give statistics both on the total number of critical points, and on newborn critical points that were generated from birth transitions during the diffusion process.
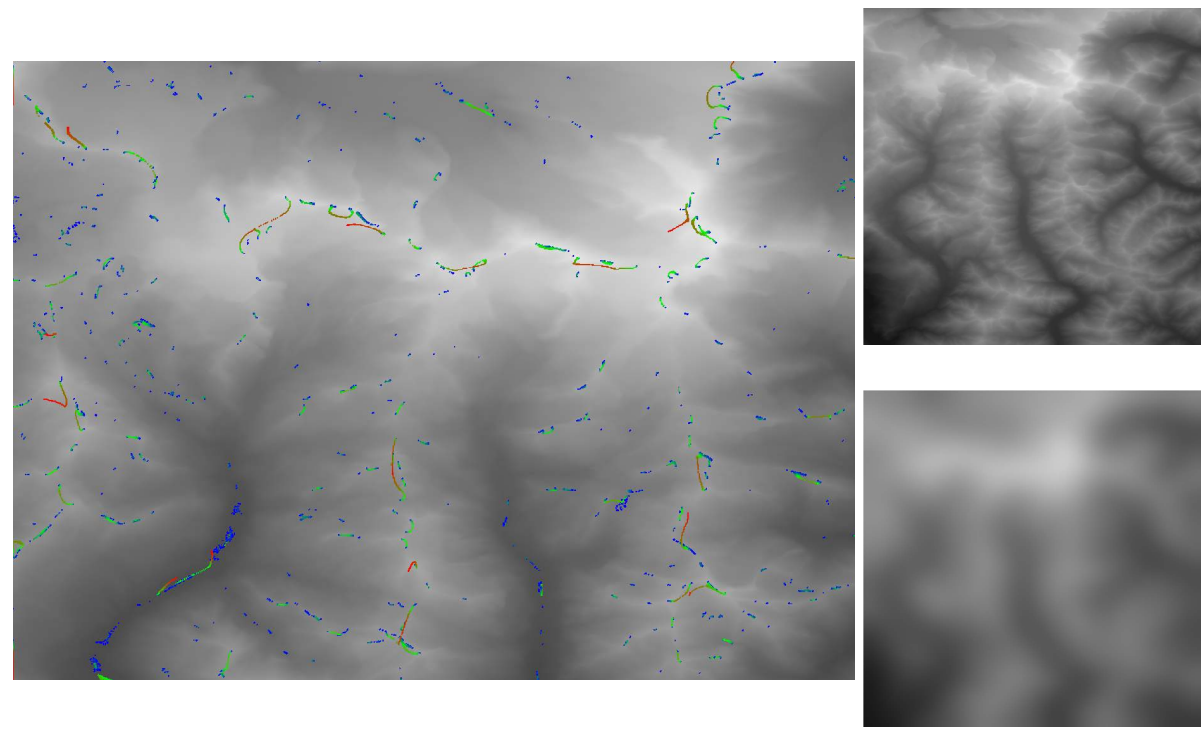

Fig. 2. Left: trajectories of all critical points on the terrain dataset (crop $900 \times 700$ pixels); color goes from blue to green to red, depending on the logarithm of time. Right: original terrain (top) and terrain filtered at scale 1024 (bottom). Zoom-in for a better view. 

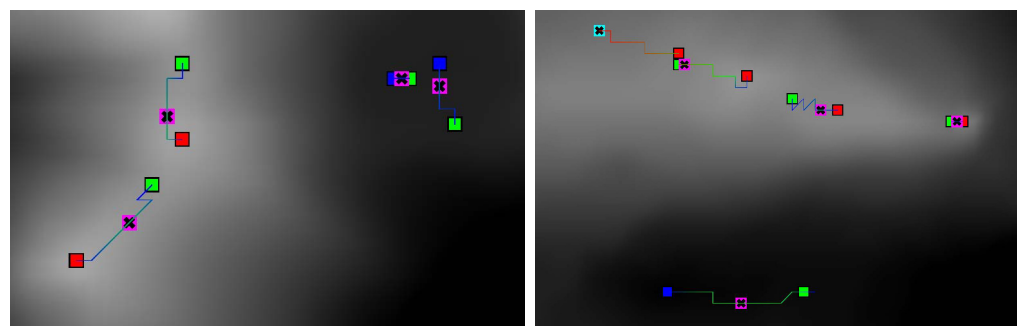

Fig. 3. Close-up of critical points trajectories on terrain (left) and on the range image (right). Markers: red maximum; blue minimum; green saddle; magenta position of collapse; cyan position of a surviving point at the largest scale.
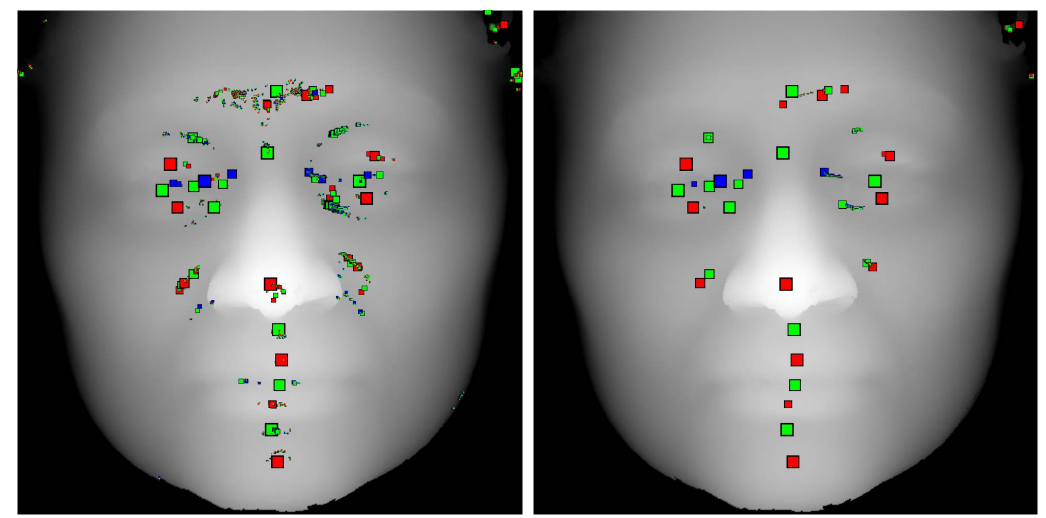

Fig. 4. Critical points alive at scale 1.0 (right) and 20.0 (left) on the range image, superimposed to the original dataset and depicted at time of their birth. Markers: blue minimum, red maximum, green saddle; size proportional to duration of life.

Our exact tracking reveals a drawback of linear scale-space, which was underestimated in previous literature: a large number of newborn critical points are generated, a few of which live long and may sometimes take the place of original features. For instance, the maximum representing the highest point of Monte Rosa Massif at large scales is not a translation of the highest peak in the original dataset, but rather a new peak born around scale 32 , while the true peak collapses short after. Substitution of features with newborn ones is hard to track, thus preventing a correct estimation of true life span in some cases. This is probably a good reason to prefer other kinds of scale-spaces, which guarantee non-creation of local extrema - one of the axioms of scale-space theory that is violated by linear scale-space in dimension larger than one. One possibility is for instance to incorporate controlled-topology filtering, as described in [4].

In figure 2 we show trajectories of points through the scale-space, superimposed to original terrain. For each point, we draw its trajectory through its life span. Time is encoded by color, ranging from blue to green to red on a 
logarithmic scale. Points do not move much until the very coarse scales. Only the few points that survive very long may undergo non-negligible displacement (reddish long trajectories) to adapt to the extreme smoothing of the signal. In Figure 3 we show close-ups of trajectories on both datasets. Note how pairs of points move toward each other and eventually collapse at different times (different colors along the lines). The marker in cyan depicts a maximum that survives up to the largest scale, in spite of being very close to a saddle in the input.

In Figure 4. we show results obtained on the range image, by querying the scale-space at scales 1 and 20 and by drawing critical points at their original positions in the input image (or at time of birth) with markers having a size proportional to the duration of life of each point. Note how several fiducial points that can be used for 3D face recognition [5] are detected by highly stable critical points. Since different fiducial points appear at different scales (e.g., centers of lips appear at a quite fine scale, while the center of the forehead appears at a very large scale), we are confident that combining information available from our scale-space model together with prior knowledge on distribution and proper scale of fiducial points, a reliable detection will be possible. We foresee that better results in this direction can be achieved by using a signal consisting of either Gaussian or total curvature of the underlying surface, rather than the raw range map. This will be the aim of our future work.

\section{Concluding Remarks}

We have presented a framework that allows representing and querying the deep structure of scale-space for the critical points of scalar functions in a continuous setting. While we have described the framework for bi-variate functions defined on a rectangle and uniformly sampled at a grid, several extensions are possible. The framework is already modular with respect to the type of scale-space, which affects just the construction of the input sequence of snapshots $\left(f_{0}, \cdots, f_{k}\right)$. Extension to irregularly sampled data, possibly on a 2-manifold domain (such as the surface of a $3 \mathrm{D}$ object), is straightforward, provided that a triangle mesh representing the domain is given. The only difference is that multiple saddles with index $k>2$ may appear. For dimensions higher than two, the mechanism can be easily adapted, by taking into account the presence of different kinds of saddles, according to Morse theory, and of related transitions.

The main limitation of the current method is that it can only track critical points of a scalar field. Other features defined as zero-crossings of differential properties could be also treated, but generalization is not always straightforward. The essential ingredient to reproduce the framework for other kinds of features is determining the events that may change the deep structure (like edge flips in our case) and to sort them by time. Once these events are found, and related transitions are defined, the rest of the framework will work unchanged.

Our analysis reveals that linear scale-space may generate many newborn features, some of which may take the place of original features, thus hindering a correct feature tracking. For this reason, we plan to incorporate controlled-topology 
filtering [4] in out future work. Integration of this mechanism comes almost for free, being also based on the analysis of the same sequence of flips.

We plan to apply our framework to the analysis of terrains and to the extraction of fiducial points for 3D face recognition. Other extensions of the framework may involve computation of the Morse complex and its evolution through scale-space, and the evaluation of topological persistence, to better characterize stability and strength of local features [11.

Acknowledgments. We wish to thank Alan C. Bovik and the Lab for Image and Video Engineering of the University of Texas for kindly providing the 3D Face Recognition Database.

\section{References}

1. Bauer, D., Peikert, R.: Vortex tracking in scale-space. In: Proceedings of the Symposium on Data Visualisation, VISSYM 2002, p. 233. Eurographics Association, Aire-la-Ville (2002)

2. Correa, C., Ma, K.L.: Size-based transfer functions: A new volume exploration technique. IEEE Trans. Visualization and Computer Graphics 14, 1380-1387 (2008)

3. de Ferranti, J.: Viewfinder panoramas (2012), http://www .viewfinderpanoramas.org/dem3.html

4. Gingold, Y.I., Zorin, D.: Controlled-topology filtering. Comput. Aided Des. 39(8), 676-684 (2007)

5. Gupta, S., Castleman, K., Markey, M., Bovik, A.: Texas 3d face recognition database. In: 2010 IEEE Southwest Symposium on Image Analysis Interpretation (SSIAI), pp. 97-100 (2010)

6. Kindlmann, G.L., Estepar, R.S.J., Smith, S.M., Westin, C.F.: Sampling and visualizing creases with scale-space particles. IEEE Trans. Visualization and Computer Graphics 15(6), 1415-1424 (2009)

7. Klein, T., Ertl, T.: Scale-space tracking of critical points in $3 \mathrm{~d}$ vector fields. In: Topology-based Methods in Visualization, Mathematics and Visualization, pp. 35-50. Springer (2007)

8. Koenderink, J.J.: The structure of images. Biological Cybernetics 50, 363-370 (1984)

9. Lindeberg, T.: Scale-Space Theory in Computer Vision. Kluwer Academic Publishers (1994)

10. Price, K.: Annotated computer vision bibliography, http://www.visionbib.com/bibliography/contents.html

11. Reininghaus, J., Kotava, N., Guenther, D., Kasten, J., Hagen, H., Hotz, I.: A scale space based persistence measure for critical points in $2 \mathrm{~d}$ scalar fields. IEEE Trans. Visualization and Computer Graphics 17(12), 2045-2052 (2011)

12. Witkin, A.P.: Scale-space filtering. In: Proc. 8th Int. Joint Conf. Art. Intell., pp. 1019-1022. Karlsruhe, Germany (1983) 